\section{A global coalition to sustain core data}

As members of an international working group to support the rapidly growing core-data resources in the life sciences, we aim to create a sustainable and accessible data infrastructure that will benefit scientists worldwide.

Although researchers have relied on international resources such as the Protein Data Bank and Flybase for decades, the current system is unsustainable because it is largely funded by short-term grants (P. E. Bourne et al. Nature 527, S16-S17; 2015). A global coalition of data resources would provide muchneeded governance structure, active service management and community-driven scientific development, which together are currently well beyond the scope of an individual investigator's typical research programme.

Science funders globally should support these data resources on the basis of their value to the research community. The coalition would define indicators to establish the core-data resources that are eligible for international support, develop models for free global access and help to assess the fraction of total research funding needed. It would also compile a set of metrics to estimate the impact, costs and benefits of each resource, including the consequences of curtailing support.

The set of data resources designated as 'core' for the life sciences would reflect a dynamic, reliable and managed portfolio that could adapt to changing scientific needs. The Global Life Sciences Data Resources Coalition will follow the lead of other international coalitions, such as those in health and physical sciences, in setting priorities and evaluating effort. (For details, see W. Anderson et al. Preprint at bioRxiv http://doi.org/b2g4; 2017). Warwick P. Anderson* Human Frontier Science Program, Strasbourg, France. wanderson@hfsp.org

${ }^{*}$ On behalf of the Global

Life Science Data Resources

Working Group (see go.nature. $\mathrm{com} / 2$ miobmk for full list).

\section{Zealandia is not a continent}

Now recognized in international law, Zealandia - the continental shelf and margin surrounding New Zealand - is vast and worthy of inquiry. However, we disagree with attempts to recharacterize it as the 'eighth continent' (see Nature http://doi. org/b2fk; 2017).

Zealandia has been extensively studied since 1895 (see

W.H.F. Smith and D. T. Sandwell Science 277, 1956-1962; 1997).

Such studies underpinned New Zealand's successful application to the United Nations to extend the limits of the continental shelf and its economic exclusion zone (see go.nature.com/2mbwmb).

Continents are defined by the Oxford English Dictionary as continuous terrestrial geographical features ("as distinguished from islands, islets, or peninsulas"). Geologists do not define continents: instead, they characterize the types of crust and delineate their geological features. By this definition, Zealandia is not a continent. Indeed, it is $94 \%$ continental margin with an extended shelf.

Elizabeth M. Dowding, Malte

C. Ebach University of New South Wales, Sydney, Australia.

dowding.e.m@gmail.com

\section{Don't compare shopping to foraging}

Economists use models of human behaviour that incorporate such non-human features as complete rationality and perfect information. Psychologists should not fall into a similar trap.

Peter Todd's article contrasts findings on shoppers' preferences for the products they know with their more exploratory behaviour in money-based laboratory experiments (see Nature 541, 294-295; 2017). The assumption in interpreting such results seems to be that consumers aim to 'optimize' the products they buy. But unless an optimal product is defined, this hypothesis is untestable because it is subjective.

Apart from perishable produce, I for one do not care about optimality. I care only about adequacy: whether an item meets my needs and is available and affordable. Once these criteria are satisfied, I need never look again. What are the chances that a new shampoo will be so much better as to be worth trying, given that my current one is adequate?

Todd's bear-foraging analogy for consumer choices therefore seems misleading. Bears presumably leave a berry patch when there is insufficient food, not because they want to find more-optimal berries. If brands appeared and disappeared like berry patches, then consumers might have to adopt a foraging strategy to sample new shampoos as they came into season.

Stephen Rader University of Northern British Columbia,

Prince George, Canada. rader@unbc.ca

\section{Vera Rubin and the darkmatter problem}

In my view, Neta Bahcall's obituary of Vera Rubin oversimplifies the dark matter problem (Nature 542, 32; 2017). Many diverse observations have contributed to the current picture, and the unknown nature of dark matter shows that this is still far from complete.

Rubin investigated rotational velocities in spiral galaxies using ionized gas regions that she was able to observe at optical wavelengths. In the 1970s, such regions could not be detected beyond a galaxy's optical image. The observed rotation curves therefore did not extend far enough to demonstrate the presence of dark matter convincingly, even though the flatness of their outer parts might convey that impression.

At that time, I and several other astronomers used the 21-centimetre radio wavelength of neutral hydrogen to determine rotation curves that often went well beyond the optical image, thereby probing the dark matter regime more effectively.

Such observations from several galaxies, coupled with optical surface photometry, permitted the calculation of local mass-to-light ratios in the outer parts of spirals (A. Bosma PhD thesis 1978; see http://go.nature. com/2mkodu5). This clinched the evidence for dark matter and still constitutes the primary source of rotation-curve data for exploring its mysteries.

Albert Bosma Marseilles Astrophysics Laboratory, France. bosma@lam.fr

\section{Stiff shirts a source of inspiration}

I was greatly saddened to read of the passing of Oliver Smithies (R. Kucherlapati Nature 542, $166 ; 2017)$. His scientific legacy will live on as an inspiration to students of all ages and levels, and their teachers (see, for example, http://smithies.lib. unc.edu).

In classes I have taught on major breakthroughs in biomedicine, Smithies' lectures were the most popular and inspirational (see, for example, the video 'Where do ideas come from?' at http://go.nature. com/2tr9e6z).

Students appreciate Smithies' points that one learns a lot from reading and that you need to do what you enjoy. And they are tickled by the story of how his inspiration for the discovery of starch gel electrophoresis came about - from watching his mother starch his father's shirts. Eric Altschuler Temple University, Philadelphia, Pennsylvania, USA. eric.altschuler@temple.edu 